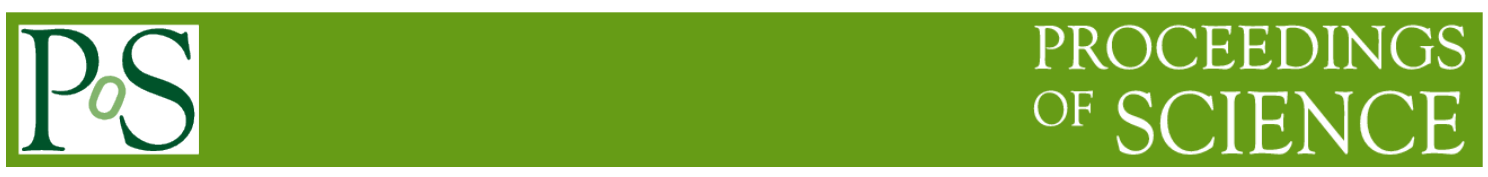

\title{
Detector R\&D for the HL-LHC upgrade
}

\section{Didier Contardo}

Université Claude Bernard Lyon 1, CNRS-IN2P3,

Institut de Physique Nucléaire de Lyon, 4 rue E. Fermi, 69622 Villeurbanne Cedex, France

E-mail: contardoein2p3. fr

The High Luminosity LHC (HL-LHC) is identified as the highest priority program for High Energy Physics, by both the European Strategy and the US Particle Physics Project Prioritization Panels [1,2]. The scientific goal is to measure precisely the properties of the Higgs boson, or of any newly discovered particles by the start of the program, and to explore the limits for new discoveries. This will require high instantaneous luminosity operation to collect about $3000 \mathrm{fb}^{-1}$ of data by the end of the program. This paper reviews the upgrades required to the ATLAS and CMS experiments to meet these challenges, along with the progress of major R\&D program to provide the appropriate technical solutions.

The European Physical Society Conference on High Energy Physics

22-29 July 2015

Vienna, Austria 


\section{LHC schedule and luminosity projections}

To fully exploit the physics potential of the LHC, CERN began planning the increase of the instantaneous luminosity before the accelerator went into operation. In the current plan, long periods of data-taking, referred to as Run 1, Run2, Run 3 etc., are interleaved with long shutdowns, designated LS1, LS2 and LS3, when the higher luminosity upgrades of the accelerator and of the experiments are accomplished. The schedule of beam operations and long shutdowns, and projections of the peak and integrated luminosities, are presented in figure 1 [3].

The modifications made to the LHC during LS1 enable to operate at a center-of-mass energy of $13 \mathrm{TeV}$, with a bunch spacing of $25 \mathrm{~ns}$. A new scheme to form the bunch trains in the Proton Synchrotron and an upgrade of the injector chain during LS2, will deliver very bright beam bunches, that should allow reaching a peak luminosity close to $2 \times 10^{34} \mathrm{~cm}^{-2} \mathrm{~s}^{-1}$ during Run 3 , providing an integrated luminosity of about $300 \mathrm{fb}^{-1}$ by 2024 .

At that time, the quadrupole magnets that focus the beams at the ATLAS and CMS collision regions are expected to be close to the end of their lives due to radiation exposure. There will be another long shutdown, LS3, to replace them with new quadrupole triplets of larger aperture and higher field, and new insertion magnets will also be installed in the section preceding this region. With these changes, the focus of the beams at the interaction point will lead to very high peak luminosities. To compensate for the beam-crossing angle, crab-cavities will be added, extending the luminous region and therefore reducing the density of p-p collisions along the beam axis.

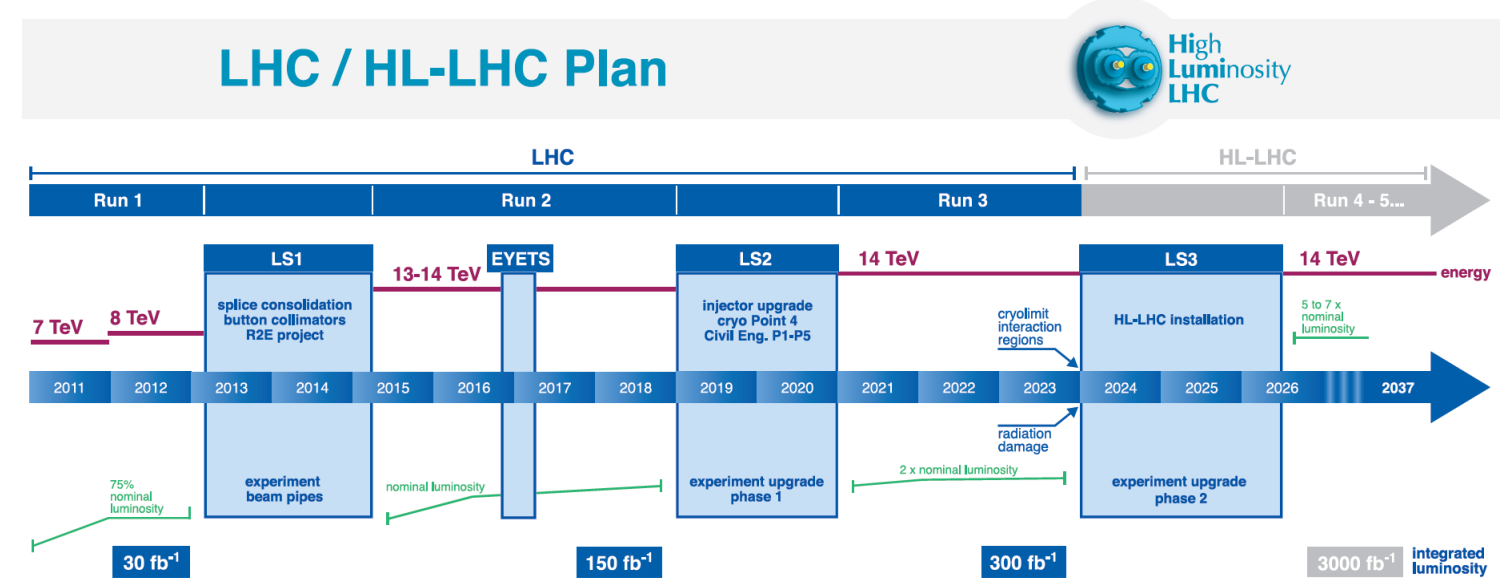

Figure 1: LHC and HL-LHC planning and luminosity projections.

The high luminosity period that follows LS3 with the upgraded LHC is referred to as HL-LHC or Phase II. The baseline operating scenario is to level the instantaneous luminosity at $5 \times 10^{34} \mathrm{~cm}^{-2} \mathrm{~s}^{-1}$ from a luminosity of $2 \times 10^{35} \mathrm{~cm}^{-2} \mathrm{~s}^{-1}$ at the beginning of the fills. Recently, an « ultimate » scenario was introduced, anticipating that a levelled luminosity of $7.5 \times 10^{34} \mathrm{~cm}^{-2} \mathrm{~s}^{-1}$ would allow increasing the integrated luminosity by about $30 \%$ with slightly shorter fills. At the baseline or ultimate luminosities, the experiments will see an average of 140 or $200 \mathrm{p}$-p collisions per beam crossing (referred to as pileup), compared to a value of 25 considered for the original detector designs. The radiation doses suffered by the detectors by the end of the program will increase by more than a factor 10 .

The ultimate instantaneous luminosity projection sets the particle occupancies, trigger requirements, and data rates that the experiments must be prepared to handle to fully exploit the potential of the accelerator. With this projection, the possibility to increase the integrated 
luminosity by $30 \%$, by the end of Phase II, sets the margin of the detector radiation tolerance to the doses accumulated in $4000 \mathrm{fb}^{-1}$.

\section{Overview of the ATLAS and CMS Phase II upgrades}

\subsection{Physics goals and requirements to the experiments}

The study of the Higgs boson will be central to the HL-LHC physics program. Nearly all of the production processes and decays will be accessible, allowing precise measurements of its couplings to other particles, and the search for rare SM and BSM decays. Figure 2 (left) shows the projection for the measurement of the Higgs boson couplings in a dataset of $3000 \mathrm{fb}^{-1}$ at $14 \mathrm{TeV}$ center-of-mass energy as a function of the vector boson or fermion masses. Compared to a precision of about $20 \%$ obtained today, percent-level precision can be reached for most coupling measurements. While no evidences of Beyond the Standard Model (BSM) phenomena were observed during Run 1, there are compelling reasons to believe that new physics must manifest at the scale of energy accessible with the LHC. If new particles are discovered during Run 2 and Run 3, the HL-LHC will be a unique facility to study their properties. The phase space of parameters covered for further discoveries will also be greatly extended. Figure 2 (right) shows an example of the mass reach for selected searches of supersymmetric particles for a dataset of $300 \mathrm{fb}^{-1}$ and $3000 \mathrm{fb}^{-1}$ at $14 \mathrm{TeV}$. Eventually, the much larger data sample will also provide access to several rare SM processes that can provide indirect evidence of new physics, or help to interpret it. More studies of the physics potential and of the performance reach at the HL-LHC can be found in references [4] and [5].
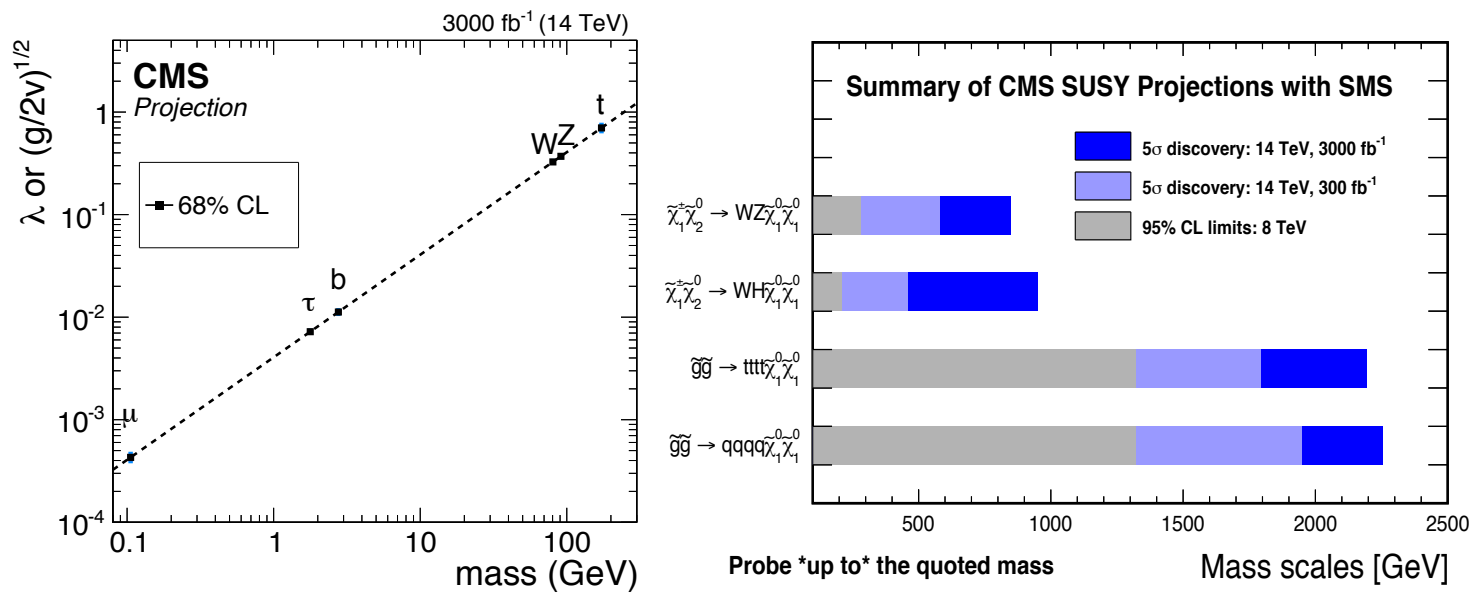

Figure 2: Projection of the Higgs boson coupling as a function of the vector boson and fermion masses with $3000 \mathrm{fb}^{-1}$ (left) and mass reach for SUSY searches in a simplified model (right) [8].

In order to achieve the physics goals, ATLAS and CMS must continue to be able to reconstruct all the standard physics analysis objects (leptons, photons, Jets (including b-quark jet tagging) and Missing Transverse Energy (MET)) with high efficiency, low fake rate, and high resolution. Preserving the current performance of all sub-detectors, in the much harsher HL-LHC environment, is therefore a primary goal for the upgrades. Because of its relatively low mass, the Higgs decay products have low energy or transverse momentum. For efficient event selection, it is therefore mandatory that the thresholds on these variables are as low as possible. This also applies to associated production processes and final states where triggering on accompanying particles can enormously reduce the level of background. As well, searches of new particles, involving long decay cascades with Dark Matter candidates, are particularly demanding for difficult hadronic and missing transverse energy trigger objects. The experiments will therefore require substantial upgrades of their data acquisition trigger systems. The Vector 
Boson Fusion (VBF) and the Vector Boson Scattering (VBS) are rare process characterized by two jets travelling in opposite directions at relatively small angles with respect to the colliding beams, with the signal products going into the more central regions of the detector. An efficient "tagging" of these jets is mandatory to enable studies of several crucial final states susceptible to reveal new physics at the HL-LHC. As the forward regions of the detectors are those suffering most from the high pileup, their performance will need to be enhanced.

\subsection{Major elements of the ATLAS and CMS upgrades}

In developing their upgrade scope, ATLAS and CMS have carried out extensive simulations to identify the sub-systems that will either not survive the radiation doses or not function efficiently because of the increased data rates. Similar radiation doses are expected in both experiments, the example of the simulated doses accumulated in CMS after $3000 \mathrm{fb}^{-1}$ is presented in figure 3. Detector longevity studies are based on the simulation of these doses and the projections of radiation effects observed in beam test measurements, special radiation exposures, and the beginning of any irradiation damage observed during Run I. These studies demonstrate that both experiments will need to replace their tracking system. For the same reason CMS will also need to replace the endcap calorimeters, while ATLAS will replace the forward calorimeter due to the high instantaneous particle flow. The design of the ATLAS and CMS upgrades are describe in details in references [6], [7], [8] and [9]. Several features and requirements that are common to the two experiments are reviewed here, without necessarily providing the specific characteristics for each detector.

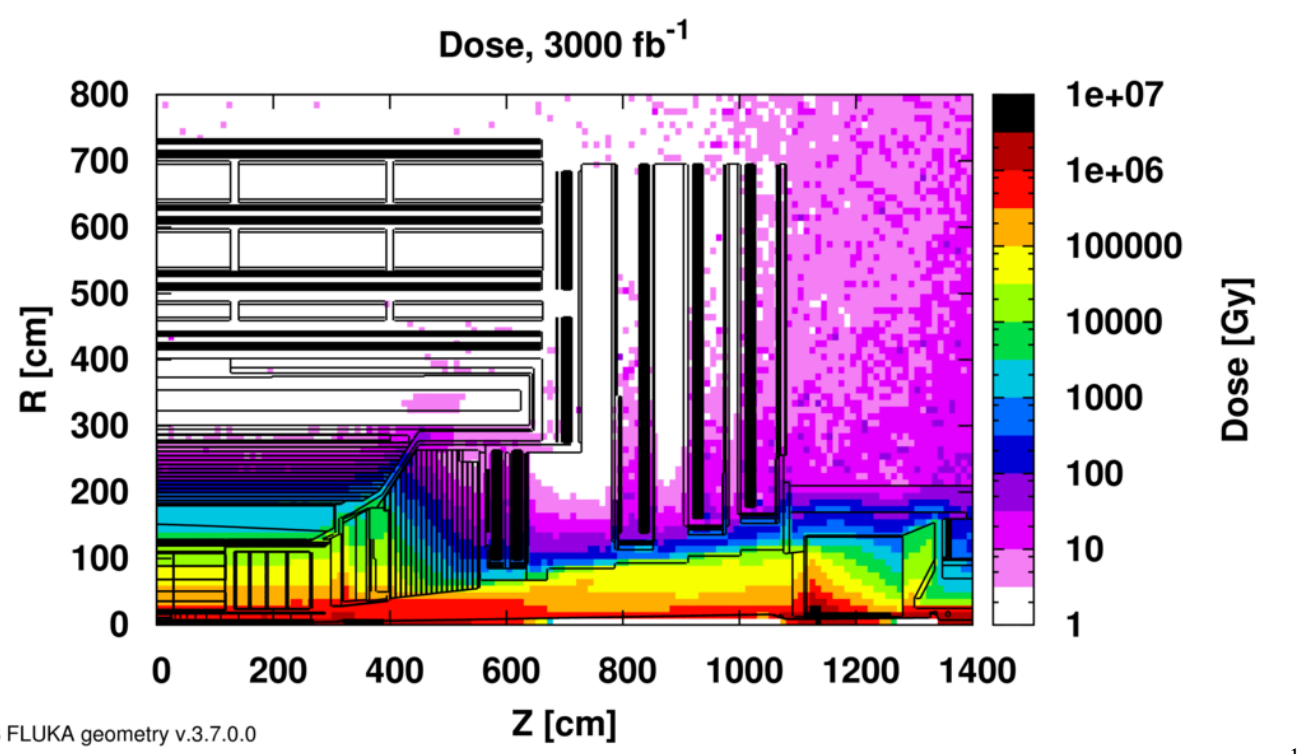

Figure 3: Absorbed dose map in the CMS detector after an integrated luminosity of $3000 \mathrm{fb}^{-1}$.

Trackers are reconstructing charge tracks and associating them to the z-position (vertex) of one of the many p-p collisions occurring at each beam crossing, with some efficiency and rate of fakes (fig. 4). They therefore provide the main mitigation of the pileup effects in the reconstruction of the hard collisions with physics interest. In both experiments the trackers will be entirely based on silicon sensors with two major systems, an outer tracker with strips and a pixel detector. Figure 5 presents a sketch of the two tracker configurations. The designs are similar, divided in ATLAS(CMS) in 5(6) barrel layers and 7(5) endcap disks, in the outer tracker, while the pixel detectors have 4 barrel layers and 12(10) endcap disks. The extension of the tracking coverage, from $\eta \simeq 2.4$ in the present detectors, to $\eta \simeq 4$, is a new crucial feature. It will allow associating the energy deposits in the calorimeters to vertices, using matching with 
the charged tracks. This will substantially improve the VBF/VBS jets identification and the missing transverse energy measurement.

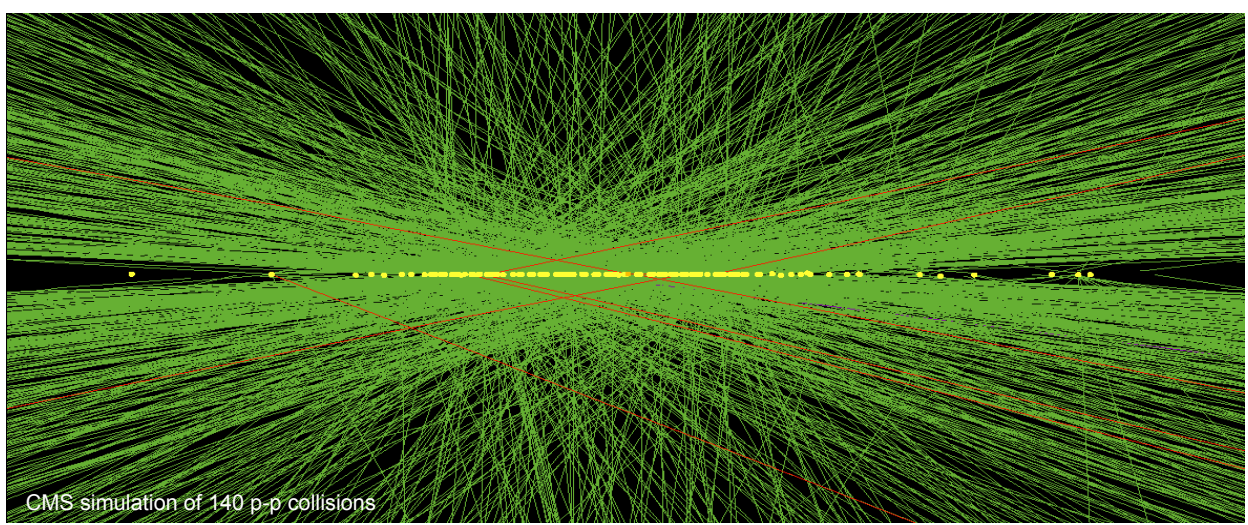

Figure 4: Simulation of the reconstruction of 140 pileup p-p collisions in the CMS tracker.

The main new feature for the pixel detectors will be the smaller size of the pixels in the range of $50 \times 50 \mu \mathrm{m}^{2}$ to $25 \times 100 \mu \mathrm{m}^{2}$ for improved resolution.

In the outer tracker the strip length will be divided by roughly a factor 4 , to about 2.5 $\mathrm{cm}$ and $5 \mathrm{~cm}$ depending on the radius, to produce similar level of occupancies as with the current detectors and operating conditions. The strip pitch will be in the range of $75 \mu \mathrm{m}$ to 90 $\mu \mathrm{m}$ to provide appropriate resolution with a binary readout. A major innovation with the new trackers will be to implement tracking information at the hardware trigger level of the experiments. The proposed trigger and data acquisition (DAQ) schemes for ATLAS and CMS are presented in figure 6 . With improved transverse momentum precision and the possibility to perform track isolation and vertex association, the background rates will be strongly reduced while maintaining the signal acceptance. In CMS, a special module design with two close sensors, with parallel strips, will allow sending hit information to the trigger at the $40 \mathrm{MHz}$ beam crossing frequency, for tracks with transverse momentum greater than $2 \mathrm{GeV}$. The hit position difference measured in a common frontend chip for the two sensors will depend on the track bending in the high magnetic field, allowing the selective readout of the proper hits. In the backend electronics (BE), the tracks will be reconstructed and fitted and then coupled with the information of other detectors to produce trigger objects for the event selection. In Atlas, information from the muon systems and the calorimeters will trigger readout of regions of interest in the tracker at $1 \mathrm{MHz}$, the tracks will then be reconstructed in the BE electronics and then matched to other detector information for the final trigger decision. ATLAS modules will feature two sensors with stereo angle for measurement of the z-coordinate, in CMS, due to the specific feature of the modules for trigger purpose, the z-measurement will be obtained in the three first layers with one of the two sensors having mini-strip (macro-pixels) of $1.5 \mathrm{~mm}$ length.


Figure 5: Configuration of the Phase II trackers in ATLAS (left) and CMS (right). 
Minimizing the material quantity in the trackers is another important characteristic to limit the multiple scattering of charged tracks and to reduce the photon conversions. The detectors will therefore use a number of new techniques developed to reduce the weights: lighter mechanical structures and materials, and new cooling and powering schemes.
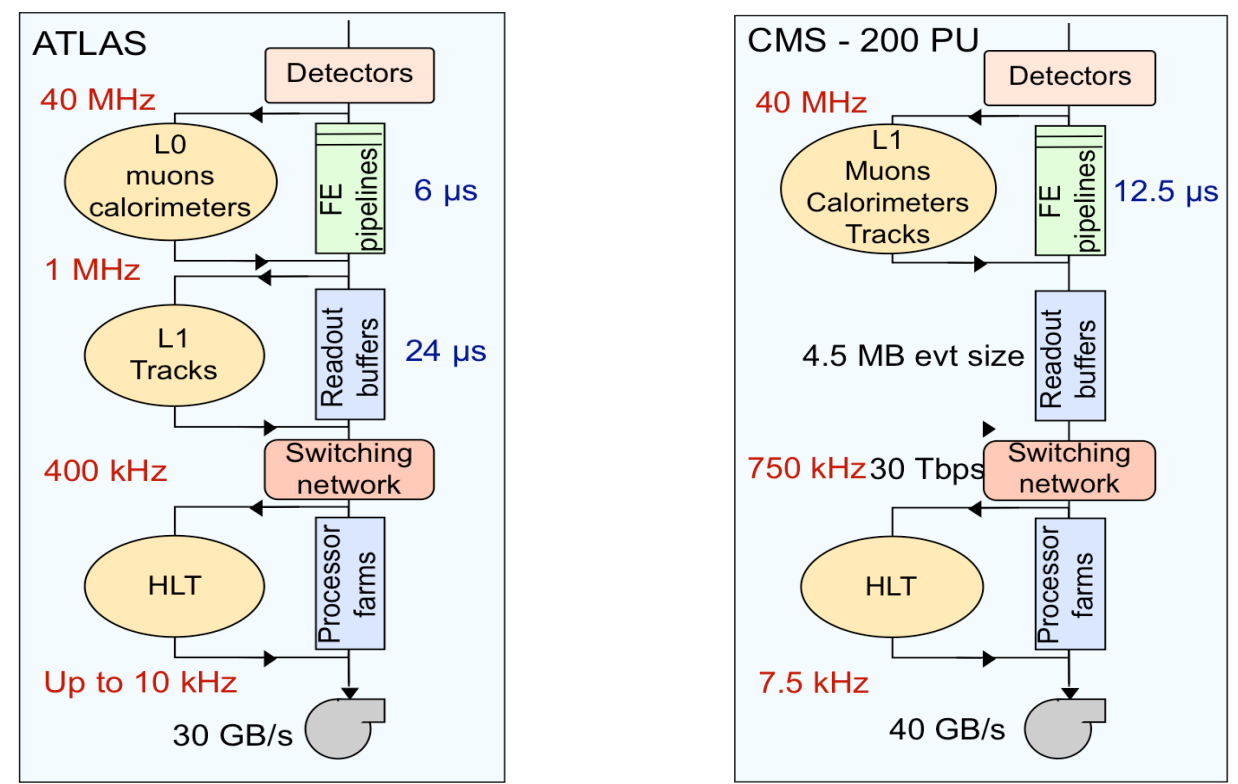

Figure 6: Schematic view of the ATLAS (left) and CMS (right) trigger/DAQ architecture.

Trigger systems will have increased latency to allow sufficient time for the hardware track reconstruction and will also have larger output rate to fully maintain the physics acceptance in all thematic areas (fig. 6). This will require the replacement of front-end and backend electronics of several calorimeter and/or muon systems that will not be replaced. The general trend for these upgrades is to profit from the bandwidth increase in data transfer devices to readout all data at $40 \mathrm{MHz}$, rather than at the trigger rate. This offers the opportunity to exploit the full granularity of the detector information at the first stage of the event selection, for improved background rejection. Increasing the trigger output rate will additionally requires additional computing power for the online (software) event selection.

Calorimeters in ATLAS and CMS are using different technologies and therefore require different upgrades. In ATLAS, the Liquid-Argon forward calorimeter covering the region $3 \lesssim \eta \lesssim 5$ will be replaced by a similar detector of about twice smaller gaps ranging from $100 \mu \mathrm{m}$ to $300 \mu \mathrm{m}$, depending on depths. It will also have a transverse granularity increased by a factor 4 in the front section. For further mitigation of pileup effects, a high granularity timing detector will be implemented in the $2.5 \lesssim \eta \lesssim 4.3$ range in front of the LAr calorimeter. Different designs and technologies are investigated for this detector to reach a timing precision of the order of tens of picoseconds. One option is a thin calorimeter with similar feature as the high granularity calorimeter foreseen for CMS. In this latter experiment, the endcap calorimeters between $1.8 \lesssim \eta \lesssim 3$ are those that will be replaced. The new system will comprise a High Granularity Calorimeter, with 28 layers of silicon sensitive elements interleaved with $\mathrm{W} / \mathrm{Cu}$ absorber to form the electromagnetic section, followed by 12 plans of silicon in brass absorber to form a first hadronic part. The hadronic energy measurement will then be completed with a scintillating tile and brass calorimeter with two depths segmentation. Additionally, both ATLAS and CMS are investigating if extending the coverage of precise timing measurement with a dedicated detection layer in front of all calorimeters could provide substantially improved mitigation of pileup, particularly for the neutral particles invisible to the trackers.

Muon systems in ATLAS and CMS will be completed with new chambers in the most difficult endcap regions, to improve the trigger capabilities and to provide redundancy to the 
existing detectors (fig. 7). Muon-tagging chambers will also be installed to extend the coverage, roughly matching the increased tracker acceptance. Both experiments will use improved conventional chambers and more recently introduced Micro-Pattern Gas Detectors (MPGD) developed by the RD51 collaboration. ATLAS will use small Thin Gap Chambers with short strips of $3.2 \mathrm{~mm}$ and $3 \mathrm{~mm}$ pitch and Micro-Megas (MM) with $0.5 \mathrm{~mm}$ pitch in the new miniwheels. CMS will use triple Gas Electron Multiplier (GE1/1, GE2/1) chambers with $140 \mu \mathrm{m}$ pitch, and low resistivity Resistive Plate Chambers (RE3/1, RE4/1) with ability for time resolution up to $100 \mathrm{ps}$ or more. The MPGD (MM and GEM) are mature developments and their installation will be anticipated during LS2, fully for ATLAS, and partially for CMS. Main R\&D for muon chambers $[4,5]$ are in: evaluation of longevity with full prototypes of present and future detectors at the new GIF++ facility at CERN; search for operating conditions minimizing aging effects: search for eco-friendly gas mixtures to replace fluoro-components; development of low resistivity RPCs with bakelite or glass; development of fabrication process for large detectors; development of multi-gap chambers; and development of improved readout electronics. A new MPGD scheme based on a well-design, that was recently introduced by the RD51 collaboration [10], is considered for the muon-tagging extensions.
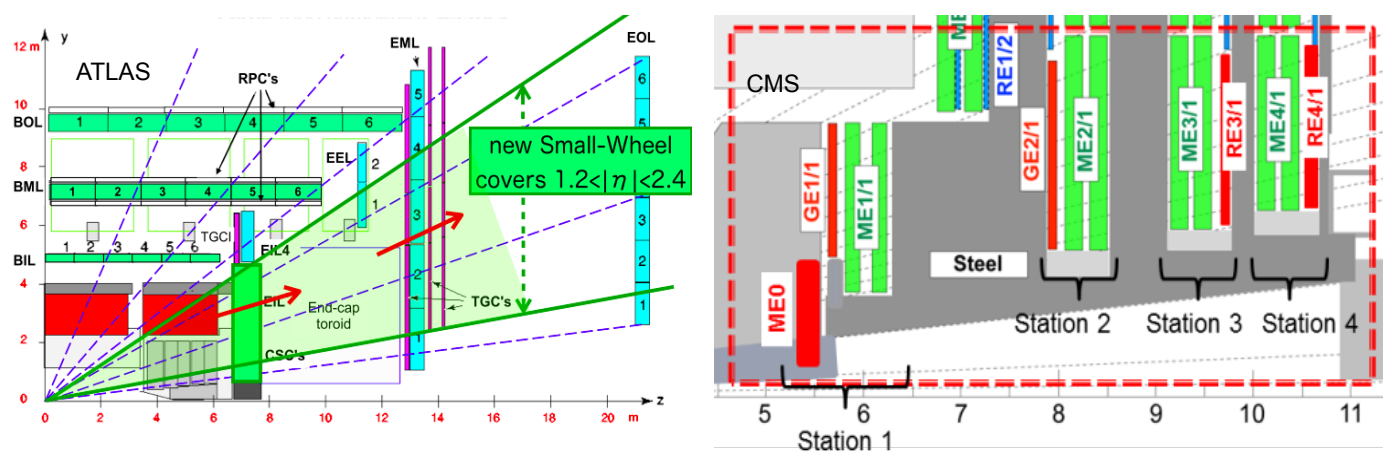

Figure 7: Transverse view of the ATLAS (left) and CMS (right) endcap muon upgrades.

\section{Main R\&D program and progresses}

\subsection{Radiation hard silicon sensors}

The development of radiation tolerant and cost effective technologies for silicon sensors is a major R\&D program for the HL-LHC experiments. This effort has largely been lead by the RD50 collaboration [11], with the development of devices with short charge collection path, either in planar or 3D technologies, and of a new generation of CMOS sensors (fig. 8). Figure 9 (left) shows the charge collected for Minimum Ionizing Particle (MIP) in planar sensors of 200 $\mu \mathrm{m}$ active thickness, as a function of the $1 \mathrm{MeV}$ equivalent neutron fluence [8]. A charge of about $10 \mathrm{ke}$ will still be collected after a fluence of $\simeq 2 \times 10^{15} \mathrm{neq} / \mathrm{cm}^{2}$, typical of the integrated dose in the first layer of the outer trackers of ATLAS and CMS after $4000 \mathrm{fb}^{-1}$. This must allow maintaining full efficiency. As well, a signal of 3 ke collected at the much higher fluence of $\simeq 2$ $\mathrm{x} 10^{16} \mathrm{neq} / \mathrm{cm}^{2}$ is deemed to be sufficient for the pixel detectors and the CMS HGC sensors; with some margins for improvements with thinner active thicknesses, lower operating temperature and higher bias voltages. The observed radiation tolerance is similar for $\mathrm{p}$-in-n or nin-p technologies and for Float-Zone (FZ) or Czochralsky (MCz) bulk materials. However, after irradiation, charge build-up appears in p-in-n sensors at the edges of the implants creating noise tails. For this reason, both CMS and ATLAS have selected the n-in-p technology for the trackers, further studies are on going for the CMS HGC where the different geometry with pads (of 0.5 $\mathrm{cm}^{2}$ to $1 \mathrm{~cm}^{2}$ ) can lead to a different behaviour. As the outer trackers of ATLAS and CMS will each require about $200 \mathrm{~m}^{2}$ of strip sensors and the CMS HGC also represents a $600 \mathrm{~m}^{2}$ area, the developments are now proceeding in close collaboration with potential vendors, able to produce large quantities of wafers. Among the possible sensor specifications, the preferred ones are for 
$\mathrm{MCz}$ material, presenting a slightly better annealing behaviour, physically thin active thickness rather than obtained from a deep diffusion process of $300 \mu \mathrm{m}$ thick sensors, and possibly 8 " rather than 6" wafers. The final specification choices will depend on the technical abilities and the costs at potential producers.
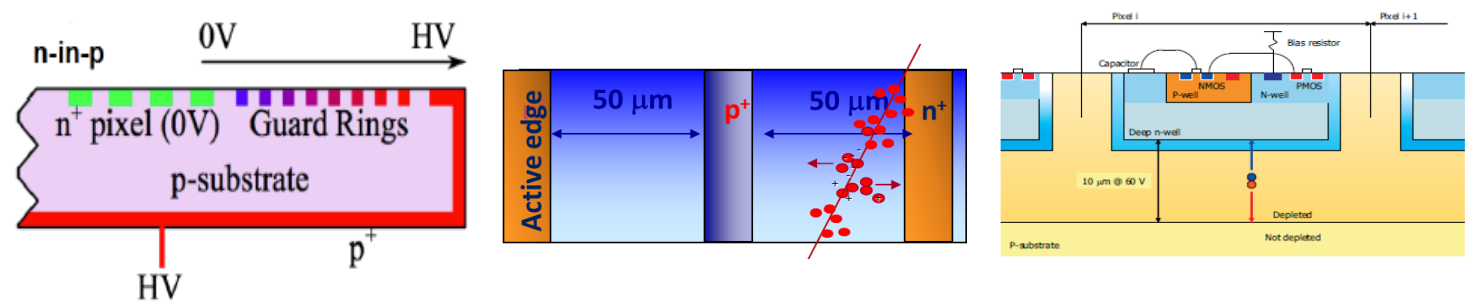

Figure 8: Sketches of planar n-in-p (left), 3D (middle), and deep n-well CMOS (right) silicon sensors.

Using 3D sensors is an alternative to the planar technology for the innermost pixel layer. They may provide more radiation tolerance margin (fig. 9 right), with the advantage of also requiring lower bias voltages [12]. However, their cost is higher and the ability to produce sensors with the small pixels foreseen needs to be established.
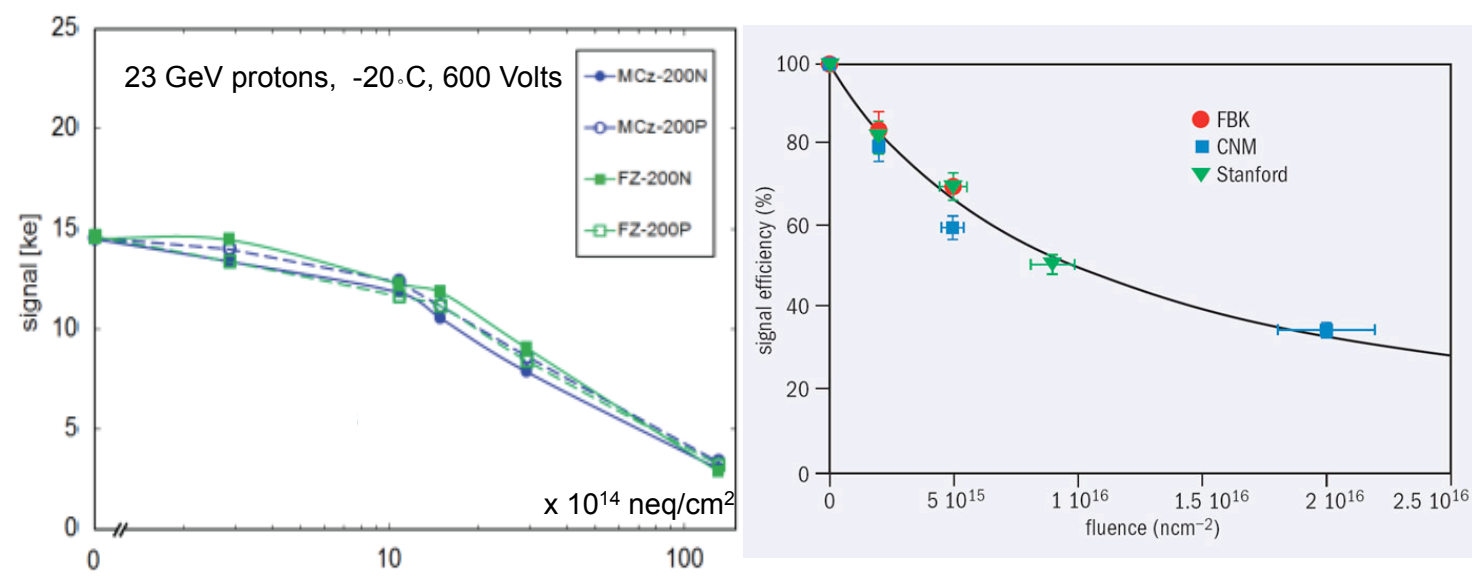

Figure 9: Charge collection in planar (left) and 3D (right) silicon sensors as a function of the accumulated $1 \mathrm{MeV}$ neutron equivalent fluence.

A third approach for both the outer tracker and the pixel detector sensors could be the HR/HV CMOS technology. The CMOS Monolithic Active Pixels (MAPs) technology has been successfully developed for low rate and radiation doses applications, offering the possibility to integrate the active part and readout of the signal in a rather standard (low-cost) commercial process. The ALICE Heavy-Ion experiment at the LHC will build the largest pixel detector ever realised using this technology [13]. To increase radiation tolerance, new processes have been developed allowing voltage biasing of the sensors. Implementing the readout features in a deep n-well (fig. 8 right) appears a promising technique that has shown good charge collection efficiency in a fluence range of $10^{15} \mathrm{neq} / \mathrm{cm}^{2}$ [14]. However, several developments would still be needed within a short timescale to produce and validate detectors that fulfil all the requirements for the HL-LHC experiments.

\subsection{Electronics systems}

Frontend ASIC chips (FE) for the readout of detectors at the HL-LHC, are of much greater complexity compared to the previous generation. Generally, the new chips must have: 
shorter amplification shaping time with lower noise; increased number of digital features; low power consumption, despite the increased channel density and the much higher data flows; and larger size in some cases. They must also be able to operate stably in very high radiation environments and after huge integrated doses. Two examples of the most complex chip characteristics are presented in Table 1. One is the Pixel chip developed by the RD53 collaboration for both ATLAS and CMS [15], and the other is the chip for the HGC calorimeter of CMS. To fulfil the requirements, the R\&D focus in recent years has been in using $130 \mathrm{~nm}$ and $65 \mathrm{~nm}$ CMOS technologies. However, the radiation hardness is not a specification of the technologies and it requires a major effort of qualification. Recent results indicate substantial sensitivity appearing in the transistor behaviour beyond doses of about 150 krads. The amplitude of the degradations depends on several design and operating parameters. Some general rules for chip designs have been derived from these studies, while investigations to identify the causes of the damage and the possible mitigations are continuing. The maximum dose sustainable by a pixel ASIC chip needs to be established compared to the cumulated dose of $1 \mathrm{Grad}$ anticipated in the innermost layer. It has to be noted that this layer represents a small fraction of the detector that is replaceable.

High bandwidth data transfer links are essential to readout the detectors at the highest possible frequency for improved off-detector event selection. $R \& D$ is in developing radiation tolerant systems with very small size and high bandwidth at low power consumption. Most, if not all, detectors in the experiments will use the GigaBitTranciever chip and the Versatile (optical) Link developed through the CERN electronics group [16]. Different version of the systems are being developed targeting a $10 \mathrm{~Gb} / \mathrm{s}$ data transmission with an $0.5 \mathrm{~W}$ power consumption. The Versatile Link will however not sustain the highest radiation doses reached in the inner pixel layers. An important R\&D is therefore needed to develop electrical links of few meters, with similar properties, to transition to a lower irradiation area. Lightness is the main challenge for these cables.

Backend electronic boards will mainly feature new backplane standards in xTCA technology with high bandwidth capabilities, and more powerful FPGAs with high bandwidth $\mathrm{I} / \mathrm{Os}$ in the range of few tens to a hundred of $\mathrm{Gb} / \mathrm{s}$. The general trend is in developing standards for generic boards that can be adapted to specific usage where required. An interesting development is in the system that will reconstruct tracks for the trigger, different options including pure FPGA techniques or usage of custom ASIC Associative Memories are being investigated.

Powering devices are crucial to minimize the quantity and volume of cables in the detectors, particularly for trackers. DC-DC Point of Load conversion or serial powering schemes will minimize the power losses. First custom versions of DC-DC converters with aircore inductor, to operate in B-field, have already been developed for the CMS pixel detector upgrade of Run 2. They can serve as a basis for more radiation tolerant and lower weight and size devices for the outer trackers, as well as for other detectors. An alternative with switched capacitors DC-DC conversion is also investigated that can have the advantage of full integration in the FE chips. For Phase II pixel detectors, the constraints of space and weight will likely require serial powering; R\&D efforts are in developing radiation tolerant solutions. 


\begin{tabular}{|c|c|}
\hline RD53 ATLAS/CMS Pixel ASIC & CMS HGC FE ASIC \\
\hline $\begin{array}{l}\text { - } \text { Noise } \simeq 1000 \mathrm{e}^{-} \\
\text {- } \text { Hit rates up to } 2-3 \mathrm{GHz} / \mathrm{cm}^{2} \\
\text { - } \text { Low power } \lesssim 1 \mathrm{~W} / \mathrm{cm}^{2} \\
\text { - } \text { Radiation tolerance to } \simeq 1 \mathrm{Grad}, 10^{16} \mathrm{n} / \mathrm{cm}^{2} \\
\text { - } \text { Trigger rate/latency up to } 1 \mathrm{MHz}, \gtrsim 10 \mu \mathrm{s} \\
\text { - Small pixel size }\left(50 \times 50 \mu \mathrm{m}^{2}\right) \\
\text { - } \text { Large chips }\left(\gtrsim 2 \times 2 \mathrm{~cm}^{2}\right)\end{array}$ & $\begin{array}{l}\text { - Shaping time } \simeq 15 \mathrm{~ns} \\
\text { - Noise } \simeq 2000 \mathrm{e}^{-}\left(\text {after } 3000 \mathrm{fb}^{-1}\right) \\
\text { - Low power } \lesssim 10 \mathrm{~mW} / \mathrm{ch}^{-} \\
\text {- } \text { Radiation tolerance } \approx 200 \mathrm{Mrad} \\
\text { - } \text { Dynamic range } 10 \mathrm{pC}-10 \mathrm{bit} \mathrm{ADC} \leq 100 \mathrm{fC} \\
\text { and Time over Threshold }(\mathrm{ToT}) \geq 80 \mathrm{fC} \text {, with } \\
\approx 50 \text { ps time resolution } \\
\text { - Channel calibration } \lesssim 1 \%\end{array}$ \\
\hline
\end{tabular}

Table 1: Example of characteristics for two major ASIC chips R\&D.

\subsection{Mechanical structures and detector services}

Light mechanical structures are crucial for trackers (fig. 10), with several constraints on their precision and thermal properties. Additionally, they will need to be stable over a large range of temperature and humidity variations, and after high accumulated radiation doses. Several R\&D are on going on materials, carbon fibres (CF) and foam compounds, light pipes in metal (Ti) or polyimide, appropriate glues, etc. New techniques to assemble or produce complex structure designs, such as 3D printing, are also being developed.
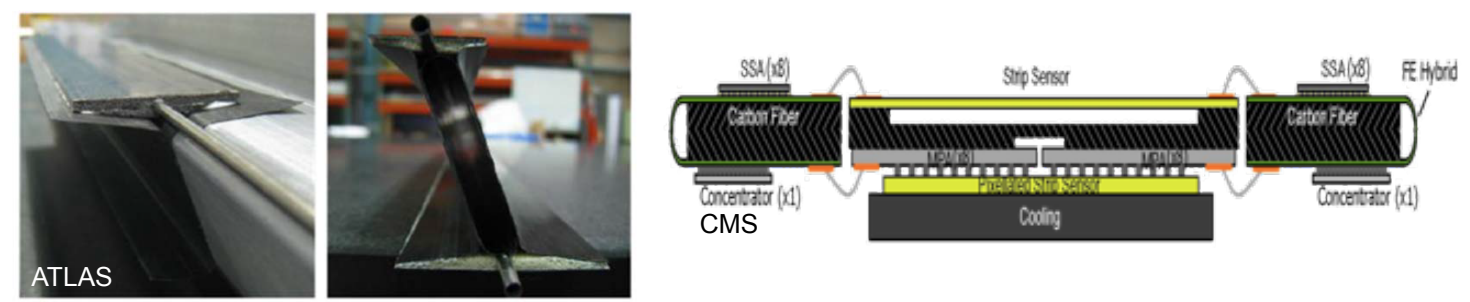

Figure 10: Low-mass CF skin and foam support structure for the ATLAS pixel detector (left) and scheme of a light (50 g) CMS module (right).

Low temperature operation is a crucial condition to control radiation damage in silicon devices. This requires development of cooling and dry atmosphere systems, and thermal shielding. The cooling systems must provide temperatures of less than $-30^{\circ} \mathrm{C}$ with high power in the range of $50 \mathrm{~kW}$. Two-phase $\mathrm{CO}_{2}$ cooling has been selected to providing the required performance, while minimizing the volume of pipes in the systems. The Phase Accumulator Controlled Loop technique, where the cold fluid is transferred from the cooling plant to the detectors, is becoming the common standard developed for the LHC experiments [17]. For distribution of the cooling to the detectors, micro-channel techniques are developed that can be directly integrated in the detectors support structure, providing improved thermal exchange while further reducing the piping material. The LHCb experiment is leading this development for the upgrade of the Silicon Vertex Locator (VELO) foreseen during LS2 [18].

\subsection{High precision timing devices}

At the HL-LHC, the rms of the luminous region longitudinally and in time are anticipated to be respectively in the range of $5 \mathrm{~cm}$ to $10 \mathrm{~cm}$ and $100 \mathrm{ps}$ to $160 \mathrm{ps}$. The precise timing measurement of charged and/or neutral particles at the scale of few tens of picoseconds could therefore provide a substantial additional handle to tracking for mitigation of the pileup effects. While there are indications that a silicon high granularity calorimeter can provide timing precision in the proper range for electromagnetic or hadronic showers, such a system will have limited coverage and may not be sufficiently efficient. $R \& D$ has therefore recently intensified in developing specific devices that can provide high timing resolution for measurement of MIPs in a more hermetic detection layer. Several new technologies are considered, among which: Multi 
Channel Plate Photo Multipliers (MCP-PMT) using a photocathode or secondary emission; Low Gain Avalanche Detectors; high gain Avalanche Photo Diodes; and Micro-Megas Chambers (fig. 11). For most of these devices, the radiation tolerance is a main issue as well as the ability to develop a large scale and low cost system. More information and resource can be found in [19].
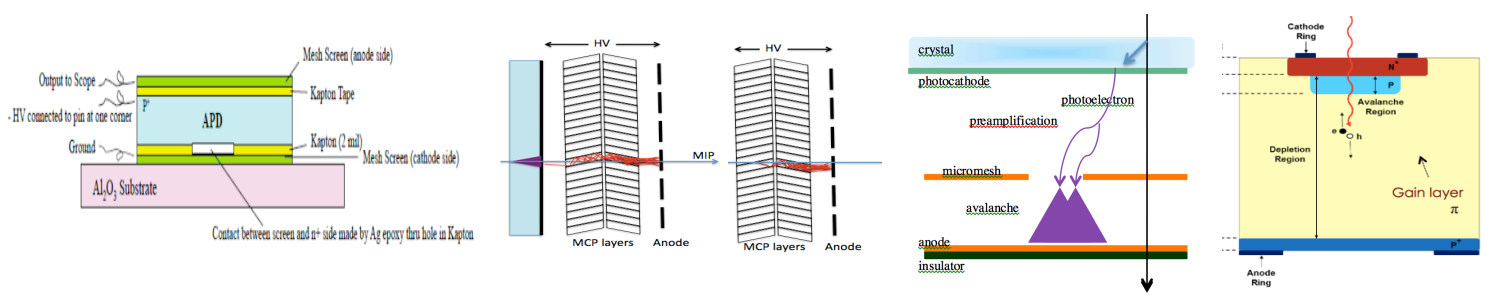

Figure 11: Schemes of high gain APD, MCP-PMT, MM and LGAD precision timing devices.

\section{Summary}

With the discovery of a Higgs boson and the recent increase of the beam energy during LS1, the LHC has greatly enhanced potential to discover new physics in the next decade. After that, the High-Luminosity LHC will be the unique facility to study properties of newly discovered particles, and to explore the limits of the parameter phase space for further discoveries. To this end, the ATLAS and CMS collaborations are developing a major program of detector upgrades to efficiently operate at the highest achievable luminosities. While several technical solutions for these upgrades have been identified, a huge R\&D effort is needed to develop final grade components and to prepare the production of the detectors.

\section{References}

[1] Physics Briefing Book. Input for the Strategy Group to draft the update of the European Strategy for Particle Physics 2013, http://europeanstrategygroup.web.cern.ch/europeanstrategygroup/Briefing_book.pdf

[2] Report of the US Particle Physics Project Prioritization Panel. http://science.energy.gov/ /media/hep/hepap/pdf/May\%202014/FINAL_P5_Report_053014.pdf

[3] HL-LHC project, http://hilumilhc.web.cern.ch/about/hl-lhc-project

[4] D. Abanneo et al., Report of the ECFA High Luminosity LHC experiments workshop, ECFA /13/284, Nov. 2013, https://cds.cern.ch/record/1631032

[5] M. Abbrescia et al., Report of the ECFA High Luminosity LHC experiments workshop, ECFA/15/289 Nov. 2014, https://cds.cern.ch/record/1983664? ln=en

[6] ATLAS Collaboration, Letter of intent for the Phase II upgrade of the ATLAS experiment, CERNLHCC-2012-022, Jan. 2013, https://cds.cern.ch/record/1502664? ln $=$ fr

[7] ATLAS Collaboration, Phase II Upgrade scoping document, CERN-LHCC-2015-020, Sept. 2015, https://cds.cern.ch/record/2055248? In=en

[8] CMS Collaboration, Technical Proposal for the Phase II upgrades of CMS, CERN-LHCC-2015010, Jun. 2015, https://cds.cern.ch/record/2020886? $\ln =e n$

[9] CMS Collaboration, the CMS Phase II Upgrade Scope Document", CERN-LHCC-2015-019, Sept 2015, https://cds.cern.ch/record/2055167? $\ln =$ en

[10] RD51 collaboration, Development of Micro-Pattern Gas Detectors, http://rd51-public.web.cern.ch/RD51-Public/Welcome.html

[11] RD50 collaboration, Radiation hard semiconductor devices for very high luminosity colliders, http://rd50.web.cern.ch/rd50/

[12] M Köhler et al., Beam Test Measurements With 3D-DDTC Silicon Strip Detectors on n-Type SubstrateIEEE, Trans. 2010, Nucl. Sci. 572987

[13] ALICE collaboration, TDR for the Upgrade of the ALICE Inner Tracking System, Nov. 2013, https://cds.cern.ch/record/1625842? $\ln =f r$

[14] W. Snoyes, ECFA HL-LHC experiments workshop, Oct. 2014, https://indico.cern.ch/event/315626/session/4/contribution/35/attachments/605603/833431/ECFA20 14CMOS WSv4.pdf 
[15] RD53 collaboration, development of pixel readout integrated circuits for extreme rate and radiation, http://rd53.web.cern.ch/RD53/

[16] P. Moreria, ECFA HL-LHC experiments workshop, Oct. 2014 , https://indico.cern.ch/event/315626/session/8/contribution/59/attachments/605633/833470/ecfa2014 moreira.pdf

[17] P. Petagna, ECFA HL-LHC experiments workshop, Oct. 2014, https://indico.cern.ch/event/315626/session/7/contribution/14/attachments/605635/833473/petagna ECFA 22oct14 final.pdf

[18] Buytaert et al., Micro channel evaporative $\mathrm{CO}_{2}$ cooling for the upgrade of the LHCb vertex detector, NIM A vol. 731, Dec 2013, 189-193

[19] T. Tabarelli de Fatis, ECFA HL-LHC experiments workshop, Oct. 2014, https://indico.cern.ch/event/315626/contribution/43/attachments/605619/833450/TTdF ECFA.pdf 